\title{
DIAGNÓSTICO PRELIMINAR DO SISTEMA DE GESTÃO DOS RESÍDUOS DE SERVIÇOS DE SAÚDE DA LIGA NORTE RIOGRANDENSE CONTRA O CÂNCER: POLICLÍNICA
}

\author{
G.S. RÊGO' \\ 'gr.giovanni@liga.org.br
}

Artigo submetido em outubro/2012 e aceito em abril/2014

DOI: 10.15628/holos.2014.1199

\section{RESUMO}

O presente artigo tem por objetivo analisar e avaliar a real situação dos resíduos de serviços de saúde gerados pela Liga Norte Riograndense Contra o Câncer: Policlínica, visando subsidiar informações para o gerenciamento correto destes resíduos, levando em consideração normas e legislações vigentes. Para atingir este objetivo, foi realizado um diagnóstico preliminar do Plano de Gerenciamento de Resíduos de Serviços de Saúde - PGRSS do ano de 2011 ao primeiro semestre de 2012. Assim, com este diagnóstico preliminar pretende-se observar o desenvolvimento e o funcionamento correto do PGRSS da Liga Norte Riograndense Contra o Câncer: Policlínica.

PALAVRAS-CHAVE: Resíduos de Serviços de Saúde, Gerenciamento, Liga Contra o Câncer.

\section{PRELIMINARY DIAGNOSIS SYSTEM OF WASTE MANAGEMENT OF HEALTH SERVICES RIOGRANDENSE NORTHERN LEAGUE AGAINST CANCER: POLYCLINIC}

\begin{abstract}
This article aims to analyze and assess the real situation of waste generated by health services Riograndense Northern League Against Cancer: Polyclinic aiming to support information for the correct management of these wastes, taking into account standardas and legislation. To achieve this goal, we conducted a
\end{abstract}

preliminary diagnosis of the waste management plan health services the year 2011 to the first half of 2012. So with this preliminary diagnosis aims to observe the development and proper functioning of PGRSS Riograndense Northern League Against Cancer: Polyclinic.

KEYWORDS: Waste Health Services, Management, League Against Cancer. 


\section{INTRODUÇÃO}

Um dos grandes desafios da atualidade é o gerenciamento dos resíduos sólidos gerados nas diversas atividades humanas: industrial, residencial, comercial, pública e serviços de saúde. Os resíduos de serviços de saúde (RSS) hospitalares é tema de interesse mundial e tem sito tratado juntamente com os problemas relativos à saúde pública. Durante esse processo diferentes materiais são utilizados, como uma grande variedade de RSS que necessitarão de um gerenciamento adequado para não prejudicar o meio ambiente.

Este gerenciamento visa minimizar a produção e proporcionar aos resíduos gerados um encaminhamento seguro e de forma eficiente, visando à proteção dos trabalhadores, a preservação da saúde pública, dos recursos naturais e do meio ambiente. Entretanto, segundo Arguello (1997) além de controlar e diminuir riscos, o gerenciamento permite elevar a qualidade e a eficiência dos serviços e saúde, sendo necessário entender que todos os membros da comunidade dos estabelecimentos de saúde, inclusive os pacientes, os visitantes e o público em geral, têm relação direta com a geração de RSS e estão igualmente expostos ao risco que tais resíduos possam acarretar.

Os RSS possuem composição variada conforme as suas características biológicas, físicas, químicas e de acordo com a origem de sua geração. Em ambiente hospitalar, destacam-se os resíduos biológicos contaminados, objetos perfurocortantes, peças anatômicas, produtos químicos, tóxicos e materiais perigosos (solventes, quimioterápicos, formaldeídos, mercúrios, dentre outros).

Portanto, com base nas características, na classificação dos grupos e no volume dos resíduos de serviços de saúde gerados, a Liga Norte Riograndense Contra o Câncer, elaborou o Plano de Gerenciamento de Resíduos de Serviços de Saúde (PGRSS) que estabelece diretrizes de manejo dos resíduos e vem a contemplar: segregação, acondicionamento, identificação, transporte interno, armazenamento intermediário, armazenamento temporário, tratamento, armazenamento externo, coleta e transporte externo e destinação final (CONAMA 1993).

O gerenciamento inadequado e a disposição desconforme de resíduos constituem fatos geradores de poluição e crimes ambientais (Naime, 2005).

A Policlínica é um hospital geral com um leque diversificado de especialidades, que vão da oncologia pediátrica à estética. A unidade possui 81 leitos para internação, centro cirúrgico com oito salas, pronto socorro, quimioterapia, ultrassonografia, raios-x e endoscopia digestiva. A unidade abriga a pediatria e a unidade de terapia intensiva da LIGA, ambas completamente equipadas e administradas por profissionais médicos especializados.

Nesse contexto e, considerando como um instrumento capaz de minimizar, ou, até mesmo impedir os efeitos adversos causados pelos RSS, sob o ponto de vista sanitário, ambiental e ocupacional, o presente artigo tem por objetivo analisar e avaliar a real situação dos resíduos de serviços de saúde gerados na Policlínica, visando subsidiar informações para o gerenciamento correto destes resíduos, levando em considerações normas e legislações vigentes. 


\section{REVISÃO BIBLIOGRÁFICA}

A preocupação com o meio ambiente aparece quando da revolução industrial sendo observado pelo homem que a poluição fabril interferia no equilíbrio da natureza, dizimando populações de flora e fauna (TACHIZAWA, 2002).

Os estudos relacionados ao meio ambiente foram intensificados a partir das últimas décadas do século XX, "entretanto definitivamente na agenda dos governos de muitos países e de diversos segmentos da sociedade civil organizada" (BARBIERI, 2004).

Os seres humanos, segundo Naime (2004), costumam despertar para uma realidade quando submetidos a situações limite, exemplo disto foi o acidente de Minamata no Japão, que pelas suas consequências catastróficas sobre o meio ambiente, despertou a consciência humana para a compreensão de que nós fazemos parte e estamos integrados em um mundo natural.

Para auxiliar no cumprimento da legislação ambiental existem muitas resoluções, portarias e outros documentos legais do Conselho Nacional de Meio Ambiente (CONAMA), Instituto Brasileiro dos Recursos Naturais Renováveis (IBAMA), Resolução da Diretoria Colegiada (RDC) da Agência Nacional de Vigilância Sanitária (ANVISA) e outros.

Entretanto, temos legislações vigentes que falam dos resíduos de serviços de saúde, que é a RDC ANVISA no 306/2004 e a Resolução CONAMA no 358/2005, que definem como geradores de resíduos de serviços de saúde todos os serviços relacionados com o atendimento à saúde humana ou animal, inclusive os serviços de assistência domiciliar e de campo.

Segundo o Manual de Gerenciamento de Resíduos de Serviços de Saúde (BRASIL, 2006) a classificação dos resíduos de saúde vem sofrendo um processo de evolução contínuo, à medida que são introduzidos novos tipos de resíduos nas unidades de saúde e como resultado do conhecimento do comportamento destes perante o meio ambiente e a saúde, como forma de estabelecer uma gestão segura com base nos princípios da avaliação e gerenciamento dos riscos envolvidos na sua manipulação.

A preocupação com o gerenciamento adequado dos resíduos gerados nas unidades de saúde tem aumentado nos últimos tempos e várias tecnologias relacionadas ao seu tratamento vêm surgindo a cada ano. A implementação de um plano de gerenciamento de resíduos de serviços de saúde apenas para cumprimento de exigências legais não resolve o problema de sua geração.

Quanto menor for a quantidade desses resíduos, menor será o custo para o seu tratamento/disposição e os problemas a eles associados; contudo, alternativas que buscam a redução da sua geração ainda são escassas (MOREIRA et al., 2005).

Acredita-se que o gerenciamento adequado dos resíduos possa contribuir significativamente para a redução da ocorrência de acidentes de trabalho, especialmente aqueles provocados por perfurocortantes. Dessa forma, também poderia ser reduzida a exposição percutânea dos trabalhadores dos serviços de saúde a materiais biológicos, uma medida no contexto da biossegurança que teria grande valor para saúde ocupacional (Garcia et al., 2004). 


\section{MÉTODO DE PESQUISA}

O presente estudo se configura como uma pesquisa exploratória e explicativa, haja vista que não só se embasou em um levantamento bibliográfico relacionado à gestão de resíduos de serviços de saúde, como também analisou criticamente os dados obtidos (SILVA; MENEZES, 2001). No estudo exploratório, buscou-se a definição do problema da pesquisa, efetuação de uma revisão de literatura em livros, periódicos, artigos e sites sobre a gestão de RSS, além da base conceitual para elaboração do instrumento de coleta de informações. No estudo explicativo, foi desenvolvido através de levantamento de informações de uma planilha de acompanhamento das saídas da bombonas de $200 \mathrm{~L}$ com peso de $25,0 \mathrm{Kg}$, onde a coleta era realizada por uma empresa licenciada pelo órgão ambiental estadual. Desta maneira, foi possível a obtenção de dados gerais do consumo de RSS realizado na unidade durante o ano de 2011 e o primeiro semestre de 2012. Para tanto, com a obtenção dos resultados, realizou-se a tabulação e construção de gráficos.

\section{ANÁLISE E DISCUSSÃO DOS DADOS}

Os RSS têm importância relevante no desenvolvimento de cada uma das fases do sistema de gerenciamento a ser implantado. Assim, para o adequado gerenciamento, intra e extra gerador, a classificação implantada na Policlínica considerou a área de geração dos resíduos, a natureza e o potencial de risco que causam a fim de oferecer segurança e minimizar riscos tanto aos colaboradores que manejam tais resíduos, quanto ao meio ambiente. A classificação permite tomar decisões quanto aos que podem ser recuperados e aos que podem seguir o fluxo para tratamento ou disposição final.

De acordo com a ANVISA os resíduos são classificados como: Grupo A: resíduos com a possível presença de agentes biológicos que, por suas características, podem apresentar riscos de infecção. Grupo B: resíduos contendo substâncias químicas que podem apresentar risco à saúde pública ou ao meio ambiente, dependendo de suas características de inflamabilidade, corrosividade, reatividade e toxicidade. Grupo C: quaisquer materiais resultantes das atividades humanas que contenham radionucleídeos em quantidades superiores aos limites de isenção especificados nas normas do CNEN e para os quais a reutilização é imprópria ou não prevista. Grupo D: resíduos que não apresentam riscos biológico, químico ou radiológico à saúde ou ao meio ambiente, podendo ser equiparados aos resíduos domiciliares. Grupo E: materiais perfurocortantes ou escarificantes.

A Policlínica gera resíduos dos grupos A, B, D e E. Os resíduos A, B e E necessitam de tratamento específico e os resíduos do grupo $D$ são destinados ao aterro sanitário do Estado.

A análise quantitativa da disposição final dos resíduos gerados na Policlínica no ano de 2011 mostra que a partir do mês de maio (figura 01) houve uma redução na quantidade dos RSS. Após o levantamento de dados de 2011, compramos neste ano o primeiro semestre de 2011 com 2012, onde se observou uma redução dos RSS gerados em 2012. Esta redução só foi possível por causa de dois indicares, o primeiro foi à contratação de um especialista em Gestão Ambiental que vem gerenciando o PGRSS e o segundo trabalhos de conscientização realizados com os colaboradores da área médica e de higienização daquela unidade. 
Resíduos Hospitalares - POL 2011/2012

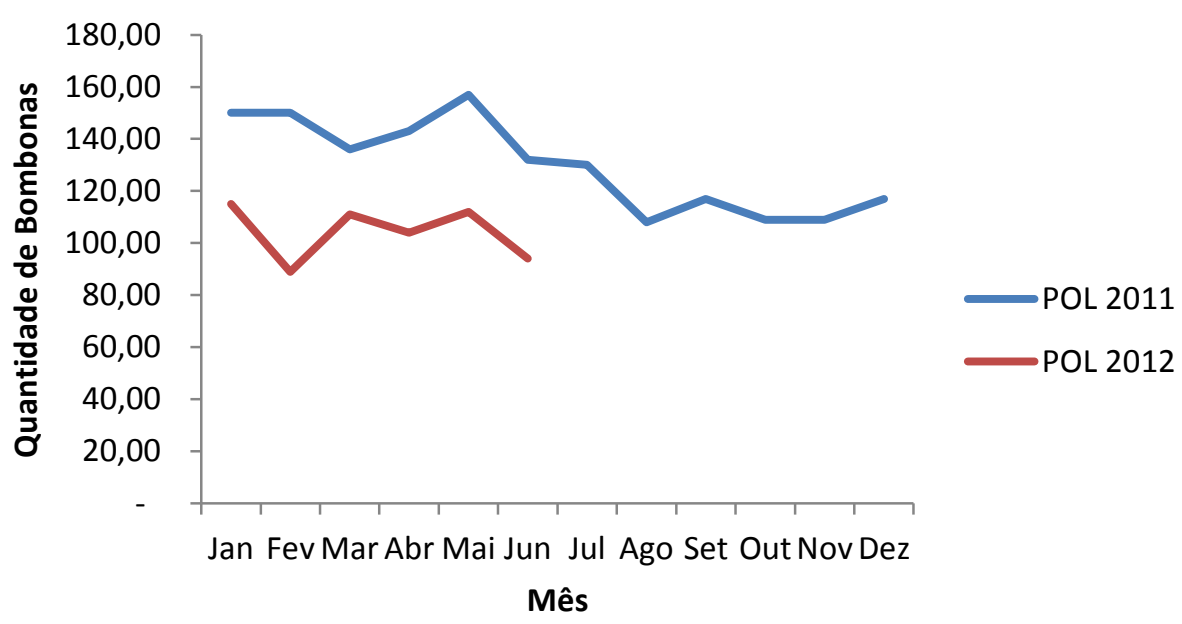

Figura 01: Quantidade de bombonas de RSS da Policlínica

\section{CONSIDERAÇÕES FINAIS}

A questão dos resíduos de serviços de saúde não pode ser analisada apenas no aspecto da transmissão de doenças infecciosas. Também está envolvida a questão da saúde do colaborador e a preservação do meio ambiente, sendo essas questões preocupações da biossegurança.

Entende-se que para ter um gerenciamento adequado à prática da educação ambiental em um ambiente privado relacionado aos resíduos de serviços de saúde não se trata, pois, somente da questão intelectual de conhecer os tipos de resíduos existentes, mas principalmente da conscientização de que é preciso mudar a forma de uso dos recursos naturais, e isto começa minimizando a geração de resíduos de serviço de saúde.

Acredita-se que este gerenciamento adequado dos resíduos possa contribuir significativamente para a redução da ocorrência de acidentes de trabalho, especialmente aqueles provocados por perfurocortantes.

Considerando o diagnóstico preliminar realizado, verificou-se que a implantação do PGRSS elaborado pela instituição, minimizou o volume dos resíduos gerados e consequentemente diminuíram os riscos de contaminação ao meio ambiente, oferecendo maior segurança a população.

\section{REFERÊNCIAS BIBLIOGRÁFICAS}

1. AGÊNCIA NACIONAL DE VIGILÂNCIA SANITÁRIA - ANVISA (Brasil). Dispõe sobre o regulamento técnico para o gerenciamento de resíduos de serviços de saúde. Resolução RDC no 306, de 07 de dezembro de 2004. D.O.U de 10 de dezembro de 2004.

2. ARGUELLO CC. Guia para o manejo interno de resíduos sólidos em estabelecimento de saúde - Centro Pan Americano de Engenharia Sanitária e Ciências do Ambiente. Brasília: Organização Pan Americana da Saúde; 1997. 
3. BARBIERI, J. C. Gestão ambiental empresarial - conceitos, modelos e instrumentos. São Paulo: Saraiva, 2004.

4. BRASIL, Ministério da Saúde. Agência Nacional de Vigilância Sanitária. Manual de gerenciamento de resíduos de serviços de saúde. Brasília: Ministério da Saúde, 2006.

5. CONAMA, Conselho Nacional de Meio Ambiente. Resolução no 5 de 05 de agosto de 1993.

6. CONAMA, Conselho Nacional de Meio Ambiente. Resolução no 358 de 29 de abril de 2005.

7. MOREIRA, C.J.; SISINNO, C. L. S. Ecoeficiência: um instrumento para a redução da geração de resíduos e desperdícios em estabelecimento de saúde. Cad. Saúde Pública, Rio de janeiro, novdez, 2005.

8. NAIME, R.; GARCIA, A. C. A. Percepção ambiental e diretrizes para compreender a questão do meio ambiente. Novo Hamburgo, Feevale, 2004.

9. NAIME, R. Gestão de resíduos sólidos. Novo Hamburgo, Feevele, 2005.

10. SILVA, E. L.; MENEZES, E. M. Metodologia da pesquisa e elaboração de dissertação. 2 a Ed. Florianópolis: Laboratório de Ensino a Distância da UFSC, 2001.

11. TACHIZAWA, T. Gestão ambiental e responsabilidade social. São Paulo: Atlas, 2002. 